\title{
Heuschnupfen ist keine Bagatellerkrankung
}

n weiten Kreisen der Bevölkerung gelten Allergien als Modekrankheiten, es ist chic, eine Allergie zu haben. Sicher gibt es Patienten, die nur relativ kurz leiden. Dies ist aber die Minderheit. Was wir Allergologen in den letzten Monaten sahen, sind z.T. schwerkranke Menschen mit stark beeinträchtigtem Allgemeinbefinden. Neben den Atemwegsbeschwerden sowie den juckenden und geröteten Augen werden nicht selten generalisierter Juckreiz, Quaddelbildung sowie Schübe von atopischem Ekzem beobachtet. In der Pollenflugzeit werden häufig Nahrungsmittelallergien manifest, die das übrige Jahr latent sind. Viele Pollinotiker klagen in den ersten Tagen kurz vor Beginn der Atemwegsbeschwerden über allgemeine Abgeschlagenheit, Kopfschmerzen und subjektives Krankheitsgefühl, bisher wissenschaftlich noch wenig untersucht und deshalb von vielen nicht ernst genommen.

Trotz aller Fortschritte in der Entwicklung von Mastzellblockern, Antihistaminika und antientzündlichen Pharmaka ist die Therapie des Heuschnupfens bei vielen Patienten immer noch schwierig. Ein systemisches Antihistaminikum hilft nur unzureichend. Die topische Therapie umfaßt nicht selten acht bis zwölf tägliche Anwendungen von Augentropfen, Nasen- und Atemsprays, was viele Patienten mit charakteristischerweise überempfindlichen Schleimhäuten schlecht tolerieren: „Jedesmal, wenn ich in die Augen tropfe oder in die Nase sprühe, muß ich an meinen Heuschnupfen denken, und die nächste Attacke geht los." Was sich in den Standardempfehlungen und Stufenschemata so schön liest, ist in der Praxis oft unbefriedigend. Bis eine Hyposensibilisierung Effekt zeigt, können Jahre vergehen. Viele Patienten und Ärzte greifen deshalb auch heute noch zum systemischen Kortison. Wir sollten dies als Allergologen nicht einfach verteufeln, sondern die Situation ernst nehmen und uns verstärkt auch um die Patienten mit dem sogenannten ,banalen Heuschnupfen“ kümmern. Wissenschaftliche Untersuchungen haben gezeigt, daß der Leidensdruck und die Beeinträchtigung der Lebensqualität bei dieser Krankheit enorm sein können.

Allergien haben immer Saison. Es sind nicht nur die Pollen, die einen allergischen Schnupfen auslösen können. Bei der ganzjährig allergischen Rhinokonjunktivitis kommt dem Innenraumklima (feuchte Wohnung) entscheidende Bedeutung zu (Brunekreef et al., S. $250 \mathrm{ff}$.)

Es ist schade, daß in der Debatte der Heuschnupfentherapie wesentlich mehr Aufmerksamkeit den seltenen Nebenwirkungen der Antihistaminika gewidmet wird als den Wirkungen (s. auch S. 230f.). Dadurch ist bei vielen Patienten große Verunsicherung entstanden. Bei sachgerechter Anwendung und Dosierung und Beachtung potentiell inkompatibler Begleitmedikation zählen nicht sedierende Antihistaminika nach wie vor zu den sichersten Arzneimitteln.

Dennoch besteht erheblicher Forschungsbedarf, um die Pollinosis wirksamer und schonender zu behandeln als dies bislang möglich ist. Wenn die Allergologengemeinschaft und die Selbsthilfegruppen seit vielen Jahren verstärkte Anstrengungen für die Allergieforschung fordern, ist es umso wichtiger, diese Forschungsarbeiten auch ernst $\mathrm{zu}$ nehmen. Dazu gehört auch die kritische Besprechung von Forschungsberichten (s. S. $233 \mathrm{ff}$. und Allergo J 1997; 6: 60-2). Es handelt sich hier nicht um eine „Leserbrief-Kampagne"; ein Forschungsbericht ist vergleichbar mit einem veröffentlichten

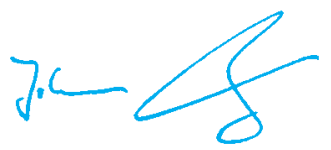

Prof. Dr. Dr. J. Ring
Buch, das gelesen und besprochen wird. Eine Buchbesprechung wird dem Autor auch nicht vor Publikation zur Stellungnahme zugestellt. Selbstverständlich kann sich aus einer solchen Buchbesprechung eine kritische Leserbrief-Diskussion entwickeln, die dem Interesse der Sache zur Klärung der offenen Fragen dient. Es geht hierbei niemals um Personen, sondern immer um die Sache. Nur die Klärung der offenen Fragen kann dazu führen, daß der leidige Streit, der seit etwa einem halben Jahr im Allergo Journal und darüberhinaus auch in anderen Medien - bis hin zu ordentlichen Gerichten - ausgetragen wurde, ein in die Zukunft gerichtetes Ende findet. Eine Arbeitsgruppe der DGAI wird in einem Konsensus die Kriterien festlegen, die offen und einer Qualitätssicherung zugänglich die für alle Interessierten vergleichbaren Bedingungen definieren, nach denen wertende Empfehlungen für Maßnahmen zur Allergenverminderung für die Betroffenen gegeben werden können.

Nach der Sommerpause stehen drei wichtige allergologische Ereignisse ins Haus (s. S. 272): der Kongreß des Ärzteverbandes Deutscher Allergologen ( ̈̈DA) in Hamburg, der Kongreß „Fortschritte der Allergologie, Immunologie und Dermatologie“, gemeinsam mit dem „Drei-Länder-Treffen der deutschen, österreichischen und schweizerischen Allergiegesellschaften" in Davos sowie der kommende Weltkongreß „International Congress of Allergology \& Clinical Immunology ICACI" in Cancun/Mexiko. Wir möchten alle allergologisch tätigen Ärzte oder an der Allergologie Interessierten auffordern, zumindest einen dieser drei Kongresse zu besuchen.

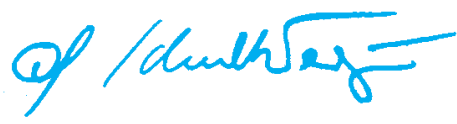

Prof. Dr. G. Schultze-Werninghaus 